\title{
PARASTATISTICS ALGEBRA, YOUNG TABLEAUX AND THE SUPER PLACTIC MONOID
}

\author{
JEAN-LOUIS LODAY AND TODOR POPOV
}

\begin{abstract}
The parastatistics algebra is a superalgebra with (even) parafermi and (odd) parabose creation and annihilation operators. The states in the parastatistics Fock-like space are shown to be in one-to-one correspondence with the Super Semistandard Young Tableaux (SSYT) subject to further constraints. The deformation of the parastatistics algebra gives rise to a monoidal structure on the SSYT which is a super-counterpart of the plactic monoid.
\end{abstract}

\begin{abstract}
à Michel
découvreur de senties inconnus où la beauté mathématique rejoint la simplicité des lois de la physique.
\end{abstract}

\section{Introduction}

Let $\mathcal{A}$ be an associative unital algebra. We denote by $\bullet$ and [, ] the associated symmetric and anti-symmetric operations:

$$
x \bullet y=x y+y x, \quad[x, y]=x y-y x .
$$

An easy lemma translates the associativity of $\mathcal{A}$ into the structure relations between the induced products.

Lemma 1.1. (M. Livernet, J.-L. Loday (unpublished)) The algebra $\mathcal{A}$ is associative

$$
x(y z)=(x y) z
$$

when the operation [, ] is a Lie bracket subject to the relations

$$
\begin{aligned}
& {[x \bullet y, z]=x \bullet[y, z]+[x, z] \bullet y \quad \text { (Leibniz rule), } .} \\
& {[[x, y], z]=x \bullet(z \bullet y)-(x \bullet z) \bullet y .}
\end{aligned}
$$

Definition 1.2. An algebra $\mathcal{P}$ is Poisson when the antisymmetric product [, ] and the symmetric product $*$ satisfy

(i) $[[x, y], z]+[[y, z], x]+[[z, x], y]=0 \quad$ (Jacobi identity)

(ii) $x *(y * z)=(x * y) * z$,

(iii) $[x * y, z]=x *[y, z]+[x, z] * y \quad$ (Leibniz rule).

Here [, ] is the Poisson bracket and $*$ stands for the commutative product of the algebra of the functions. 
A straightforward corollary of the Lemma (1.1) is the following one

Corollary 1.3. Let the associative algebra $\mathcal{A}$ be also a Poisson algebra with commutative product $*$ coinciding with the symmetric product: $\bullet=*$. Then the associativity of • implies

$$
[[x, y], z]=0 .
$$

\section{Parastatistics algebra}

The relations of type (11) appear in the general quantization scheme due to H.S. Green [9]. Instead of the canonical anticommutation relations between the creation $a_{i}^{\dagger}$ and annihilation $a_{j}$ modes of the fermions

$$
\left[a_{i}, a_{j}^{\dagger}\right]_{+}=\delta_{i j}, \quad\left[a_{i}, a_{j}\right]_{+}=0, \quad\left[a_{i}^{\dagger}, a_{j}^{\dagger}\right]_{+}=0,
$$

Green introduced a scheme coined parafermi quantization based on the exchange relations

$$
\begin{aligned}
& {\left[\left[a_{i}^{\dagger}, a_{j}\right], a_{k}^{\dagger}\right]=2 \delta_{j k} a_{i}^{\dagger} \quad\left[\left[a_{i}^{\dagger}, a_{j}\right], a_{k}\right]=-2 \delta_{i k} a_{j}} \\
& {\left[\left[a_{i}^{\dagger}, a_{j}^{\dagger}\right], a_{k}^{\dagger}\right]=0 \quad\left[\left[a_{i}, a_{j}\right], a_{k}\right]=0}
\end{aligned}
$$

The associative algebra having relations (3) will be referred to as creation-annihilation parafermi algebra. The bilinear canonical relations imply the trilinear parafermi relations, (2) $\Rightarrow(3)$ thus the canonical quantization is a particular example of paraquantization.

The creation (and the annihilation) parafermi modes alone close a subalgebra with double commutator relations as in (11).

More generally, for a system including both odd and even degrees of freedom, the parastatistics relations

$$
\begin{aligned}
& \llbracket \llbracket a_{i}^{\dagger}, a_{j} \rrbracket, a_{k}^{\dagger} \rrbracket=2 \delta_{j k} a_{i}^{\dagger} \quad \llbracket a_{k}, \llbracket a_{j}^{\dagger}, a_{i} \rrbracket \rrbracket=2 \delta_{j k} a_{i} \\
& \llbracket \llbracket a_{i}^{\dagger}, a_{j}^{\dagger} \rrbracket, a_{k}^{\dagger} \rrbracket=0 \quad \llbracket a_{k}, \llbracket a_{j}, a_{i} \rrbracket \rrbracket=0
\end{aligned}
$$

define the superalgebra where $\llbracket x, y \rrbracket:=x y-(-1)^{\hat{x} \hat{y}} y x$ is a Lie superbracket, the parabose operators are odd, and the parafermi ones are even generators (note that here the grading is the opposite to the usual one in which bose are even and fermi are odd). The Lie superalgebra $\mathcal{L}$ closed from the creation parastatistics modes $a_{i}^{\dagger}$ is 2nilpotent in view of the relation $\llbracket \llbracket a_{i}^{\dagger}, a_{j}^{\dagger} \rrbracket, a_{k}^{\dagger} \rrbracket=0$, cf. (4), thus for the Lie superalgebra $\mathcal{L}$ we have

$$
\mathcal{L}=V \oplus \llbracket V, V \rrbracket .
$$

Definition 2.1. Let us denote by $V$ the vector superspace of dimension $m \mid n$ spanned by the even $(\hat{i}=0)$ parafermionic and odd $(\hat{i}=1)$ parabosonic creation operators $V=$ $V_{0} \oplus V_{1} \cong \mathbb{C}^{m \mid n}$ and we suppose $V_{0}=\bigoplus_{i=1}^{m} \mathbb{C} a_{i}^{\dagger}$ and $V_{1}=\bigoplus_{i=m+1}^{m+n} \mathbb{C} a_{i}^{\dagger}:=\bigoplus_{i=\overline{1}}^{\bar{n}} \mathbb{C} a_{i}^{\dagger}$.

The creation parastatistics algebra $P S(V)$ is the universal enveloping algebra of the Lie superalgebra $\mathcal{L}$,

$$
P S(V)=U(\mathcal{L})=T(V) / I(V) \quad I(V)=\left(\llbracket V, \llbracket V, V \rrbracket_{\otimes} \rrbracket_{\otimes}\right)
$$

that is, the factor of the tensor algebra $T(V)$ by the ideal $I(V)$ generated by the double supercommutators [8]. 
From the Poincaré-Birkhoff-Witt theorem for Lie superalgebras one gets $[8$

$$
P S(V)=U(\mathcal{L}) \cong \mathbb{S}(V) \otimes \mathbb{S}(\llbracket V, V \rrbracket)
$$

where $\mathbb{S}(A)$ is the symmetric superalgebra generated from $A$ (see below).

2.2. Parastatistics Fock space. Palev shown [20] that the creation-annihilation superalgebra (4) with $m$ parafermionic and $n$ parabosonic degrees of freeedom is isomorphic to the the orthosymplectic superalgebra $\mathfrak{o s p}_{1+2 m \mid 2 n}$. This isomorphism allows us to define the parastatistics Fock space as a special $U\left(\mathfrak{o s p}_{1+2 m \mid 2 n}\right)$-representation.

Definition 2.3. The representation of the universal enveloping algebra $U\left(\mathfrak{o s p}_{1+2 m \mid 2 n}\right)$ built on a unique vacuum space $|0\rangle$ such that

$$
a_{i}|0\rangle=0 \quad \llbracket a_{i}, a_{j}^{\dagger} \rrbracket|0\rangle=p \delta_{i j}|0\rangle
$$

will be referred to as parastatistics Fock space $\mathcal{F}(m \mid n ; p)$ of the creation-annihilation algebra (4) with $m$ parafermions and $n$ parabosons. The number $p$ is called the order of the parastatistics.

The creation parastatistics algebra PS(V) is universal in the following sense; the parastatistics Fock space $\mathcal{F}(m \mid n ; p)$ of order $p$ is isomorphic to the quotient

$$
\mathcal{F}(m \mid n ; p) \cong P S(V) / M(V, p) .
$$

For $p=1$ the parastatistics Fock space $\mathcal{F}(m \mid n ; p)$ is the ordinary Fock space $\mathcal{F}$ of a system with $m$ fermions and $n$ bosons.

Lemma 2.4. The elements $E_{i j}=\frac{1}{2} \llbracket a_{i}^{\dagger}, a_{j} \rrbracket$ of the creation-annihilation algebra (4) satisfy

$$
\llbracket E_{i j}, E_{k l} \rrbracket=E_{i l} \delta_{j k}-(-1)^{(\hat{i}-\hat{j})(\hat{k}-\hat{l})} E_{j k} \delta_{i l}
$$

i.e. they close the general linear Lie superalgebra $\mathfrak{g l}_{m \mid n}$. The superspace $V$ is a fundamental representation of the superalgebra $\mathfrak{g l}_{m \mid n}, E_{i j} a_{k}^{\dagger}=\delta_{j k} a_{i}^{\dagger}$.

The algebra $\mathfrak{g l}_{m \mid n}$ can be extended to the parabolic subalgebra

$$
\mathcal{P}=\operatorname{span}\left\{\llbracket a_{i}^{\dagger}, a_{j} \rrbracket, a_{i}, \llbracket a_{i}, a_{j} \rrbracket ; i, j=1, \ldots, m+n\right\}
$$

thus we have the chain of inclusions $\mathfrak{g l}_{m \mid n} \subset \mathcal{P} \subset \mathfrak{o s p}_{1+2 m \mid 2 n}$. The subalgebra $\mathcal{P}$ acts trivially on the vacuum space $\mathbb{C}|0\rangle$ hence the parastatistics Fock space $\mathcal{F}(m \mid n ; p)$ is the induced module

$$
\mathcal{F}(m \mid n ; p)=\operatorname{Ind}_{\mathcal{P}}^{\mathfrak{o s p}_{1+2 m \mid 2 n}} \mathbb{C}|0\rangle
$$

The inclusion $\mathfrak{g l}_{m \mid n} \subset \mathfrak{o s p}_{1+2 m \mid 2 n}$ implies that the space $\mathcal{F}(m \mid n ; p)$ has decomposition into irreducible representations of $\mathfrak{g l}_{m \mid n}$.

The tensor powers $V^{\otimes r}$ of the vector representation $V$ are completely reducible $U\left(\mathfrak{g l}_{m \mid n}\right)$-modules. The $U\left(\mathfrak{g l}_{m \mid n}\right)$-irreducible subrepresentations of $V^{\otimes r}$ are indexed by Young diagrams (or partitions), i.e., in the same vein as the representations of the symmetric group.

The roots of such a parallel of representations are in the double centralizing property of the superalgebra action and the sign permutation action of $\mathfrak{S}_{r}$ in $\operatorname{End}\left(V^{\otimes r}\right)$. 
Theorem 2.5. (The Schur-Weyl duality [3]) Let the $\mathfrak{g l}_{m \mid n}$-action $\rho$ on $V^{\otimes r}$ be

$$
\rho(X)\left(a_{i_{1}}^{\dagger} \otimes \ldots \otimes a_{i_{r}}^{\dagger}\right):=\sum_{k}(-1)^{p_{k}(X)} a_{i_{1}}^{\dagger} \otimes \ldots\left(X a_{i_{k}}^{\dagger}\right) \ldots \otimes a_{i_{r}}^{\dagger}, \quad X \in \mathfrak{g l}_{m \mid n}
$$

where $p_{k}(X)=\hat{X} \sum_{j=k+1}^{r} \hat{i}_{j}$. Let the sign permutation action $\sigma$ on $V^{\otimes r}$ be

$$
\left(a_{i_{1}}^{\dagger} \otimes \ldots \otimes a_{i_{r}}^{\dagger}\right) \sigma(\tau):=\epsilon(\tau, I) a_{\tau^{-1}\left(i_{1}\right)}^{\dagger} \otimes \ldots \otimes a_{\tau^{-1}\left(i_{r}\right)}^{\dagger}, \quad \tau \in \mathfrak{S}_{r}
$$

where $\epsilon(\tau, I)= \pm 1$ is the parity of the odd-odd (paraboson) exchanges. The actions $\rho$ and $\sigma$ of the generators are extended by linearity. The algebras $\sigma\left(\mathbb{C}\left[\mathfrak{S}_{r}\right]\right)$ and $\rho\left(U\left(\mathfrak{g l}_{m \mid n}\right)\right)$ are centralizers to each other in $\operatorname{End}\left(V^{\otimes r}\right)$

$$
\rho\left(U\left(\mathfrak{g l}_{m \mid n}\right)\right)=\operatorname{End}_{\mathfrak{S}_{r}}\left(V^{\otimes r}\right) \quad \sigma\left(\mathbb{C}\left[\mathfrak{S}_{r}\right]\right)=\operatorname{End}_{U\left(\mathfrak{g l}_{m \mid n}\right)}\left(V^{\otimes r}\right) .
$$

Thus the superalgebra modules are determined from those of $\mathfrak{S}_{r}$. An irreducible $\mathfrak{S}_{r}$-module $S^{\lambda}$ defines an irreducible $U\left(\mathfrak{g l}_{m \mid n}\right)$-module $V^{\lambda}$ through the Schur functor

$$
\mathbb{S}^{\lambda} V=V^{\lambda}:=S^{\lambda} \otimes_{\mathfrak{S}_{r}} V^{\otimes r}
$$

where $\mathfrak{S}_{r}$ acts on $V^{\otimes r}$ by the sign permutation action $\sigma$. For instance the $r$-th degree of the symmetric algebra $S(V)$ is the Schur module attached to a single row diagram with $r$ cells

$$
S(V)=\oplus_{r \geq 0} \mathbb{S}^{r} V, \quad \mathbb{S}^{r} V=V^{(r)} \quad\left(\mathbb{S}^{0} V:=\mathbb{C}\right) .
$$

The map $\sigma: \mathbb{C}\left[\mathfrak{S}_{r}\right] \rightarrow \operatorname{End}_{U(\mathfrak{g r}(V))}\left(V^{\otimes r}\right)$ is known to be surjective and its cokernel is given by Berele and Regev Hook lemma.

Theorem 2.6. (Berele and Regev [3]) The image $\sigma\left(\mathbb{C}\left[\mathfrak{S}_{r}\right]\right)=\bigoplus_{\lambda \in \Gamma} A^{\lambda}$ of the sign representation $\sigma$ in $\operatorname{End}\left(V^{\otimes r}\right)$ for the $m \mid n$-dimensional vector representation $V$ is labelled by the subset $\Gamma$ of diagrams with $r$ cells included in a hook of arm-height $m$ and leg-width $n$,

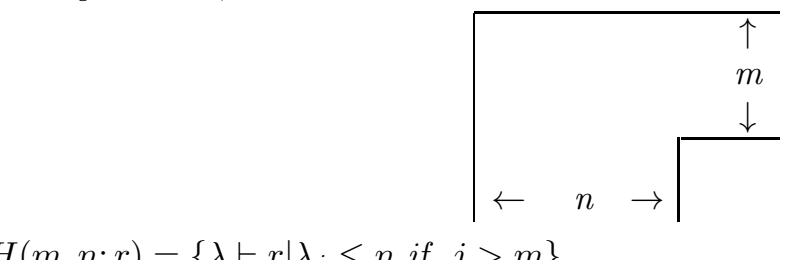

$$
\sigma\left(\mathbb{C}\left[\mathfrak{S}_{r}\right]\right) \cong \bigoplus_{\lambda \in H(m, n ; r)} S^{\lambda}
$$

Definition 2.7. The super semistandard Young tableaux or $(m, n)$-semistandard Young tableaux of shape $\lambda$ are the fillings of the Young diagram $\lambda$ with the letters of the ordered signed alphabet $\{1, \ldots, m, \overline{1}, \ldots, \bar{n}\}$ such that the even indices are nondecreasing in rows and increasing in columns whereas the odd indices are increasing in rows and nondecreasing in columns.

We denote by $H(m, n)=\bigcup_{r} H(m, n ; r)$ all the diagrams within the hook. Note that a $(m, n)$-semistandard Young tableau is always of shape within the hook $H(m, n)$, $\lambda \in H(m, n)$. 
The basis of the irreducible $\mathfrak{g l}_{m \mid n}$-module $V^{\lambda}$ is indexed by the super semistandard Young tableau of shape $\lambda \in H(m, n)$. As $\mathbb{C}\left[\mathfrak{S}_{r}\right]-U\left(\mathfrak{g l}_{m \mid n}\right)$-bimodule the superspace $V^{\otimes r}$ is isomorphic to

$$
V^{\otimes r} \cong \bigoplus_{\lambda \in H(m, n ; r)} S^{\lambda} \otimes V^{\lambda}
$$

The irreducible $\mathfrak{g l}_{m \mid n^{-m o d u l e}} V^{\lambda}$ can be lifted to a module of the supergroup $G L(m \mid n)$ [10], thus $V^{\lambda}$ can also be referred to as linear supergroup $G L(V)$-module.

The Weyl theorem for the polynomial $G L(m)$-modules is a particular instance of the Hook theorem when $V=V_{0}$ is a (bosonic) vector space, since $H(m, 0)$ are the diagrams with no more than $m$ rows. The basis of an irreducible $G L(m)$-module $V^{\lambda}$ with $\lambda_{m+1}=0$ is labelled by the usual semistandard Young tableaux (with indices nondecreasing in rows and increasing in columns) of shape $\lambda$.

It is worth noting that $H(0, n)$ are the diagrams within a vertical strip, i.e., with no more than $n$ columns, which label the $G L(0 \mid n)$-modules when $V$ is a fermionic space $V=V_{1}$. The isomorphism $G L(0 \mid n) \cong G L(n)$ put in correspondence a Young tableau to its transposed.

\section{The $U\left(\mathfrak{g l}_{m \mid n}\right)$-Module $P S(V)$ And The $\mathfrak{S}$-Module $P S$}

Lemma 3.1. The double supercommutator subspace $I_{3}(V)=\llbracket V, \llbracket V, V \rrbracket_{\otimes} \subset V^{\otimes 3}$ is an irreducible Schur module

$$
V^{(2,1)}=I_{3}(V)=e \mathbb{C}\left[\mathfrak{S}_{3}\right] \otimes_{\mathfrak{S}_{3}} V^{\otimes 3}
$$

arising as the Schur functor image of the $\mathfrak{S}_{3}$-module $I(3)=e \mathbb{C}\left[\mathfrak{S}_{3}\right] \cong S^{(2,1)}$, where e stands for the Eulerian idempotent [16]

$$
e=\frac{1}{3}\left(123-\frac{1}{2}(231+213+132+312)+321\right) .
$$

Proof. The cyclic permutation of $I_{3}(V)$ vanishes due to the super Jacobi idenity

$$
\llbracket x, \llbracket y, z \rrbracket \rrbracket+(-1)^{\hat{x} \hat{y}+\hat{x} \hat{z}} \llbracket y, \llbracket z, x \rrbracket \rrbracket+(-1)^{\hat{x} \hat{z}+\hat{y} \hat{z}} \llbracket z, \llbracket x, y \rrbracket \rrbracket=0, \quad x, y, z \in V
$$

thus $I_{3}(V) \cap V^{\left(1^{3}\right)}=0=I_{3}(V) \cap V^{(3)}$ and, counting the dimensions, we conclude that $I_{3}(V)=V^{(2,1)}$. For the $\mathfrak{S}_{3}$-representation $I(3)$ the Jacobi identity implies

$$
I(3)=\operatorname{Ind}_{\mathbb{Z}_{3}}^{\mathfrak{S}_{3}} \mathbb{1} \text {. }
$$

The rest is a direct calculation.

Theorem 3.2. Let $V$ be the $m \mid n$-dimensional super space. In the decomposition of the $U\left(\mathfrak{g l}_{m \mid n}\right)$-module $P S(V)$ into irreducibles each $V^{\lambda}, \lambda \in H(m, n)$ appears once and exactly once $P S(V) \cong \bigoplus_{\lambda \in H(m, n)} V^{\lambda}$.

Proof. Let us consider first the case of an $m$-dimensional even space $V=V_{0}$. The left hand side of the Schur formula

$$
\prod_{i=1}^{m} \frac{1}{1-x_{i}} \prod_{1 \leq i<j \leq m} \frac{1}{1-x_{i} x_{j}}=\sum_{\lambda} s_{\lambda}(x)
$$

is the character of the $U\left(\mathfrak{g l}_{m}\right)$-module $P S(V) \cong S(V) \otimes S([V, V])$ in view of the Poincaré-Birkhoff-Witt theorem. Then the sum of the Schur polynomials $s_{\lambda}(x)$ (which 
are characters of the irreducible $U\left(\mathfrak{g l}_{m}\right)$-modules $\left.V^{\lambda}\right)$ on the right hand side implies $P S(V) \cong \bigoplus_{\lambda} V^{\lambda}=\bigoplus_{\lambda \in H(m, 0)} V^{\lambda}$ for $V=V_{0}$ where the sum on $\lambda$ runs on the Young diagrams with no more than $m$ rows, $\lambda_{m+1}=0$. Thus all nontrivial $U\left(\mathfrak{g l}_{m}\right)$-modules modules are present in $P S\left(V_{0}\right)$.

Lemma 3.3. Let us have $\mathfrak{S}=\bigoplus_{r>0} \mathfrak{S}_{r}$. The decomposition of the $\mathfrak{S}$-module PS= $\bigoplus_{r \geq 0} P S(r)$ contains each irreducible finite dimensional $\mathbb{C}\left[\mathfrak{S}_{r}\right]$-module $S^{\lambda}, r \geq 0$, exactly once $P S=\bigoplus_{\lambda} S^{\lambda}$.

Proof of the lemma. We have $P S\left(V_{0}\right)=\bigoplus_{r \geq 0} P S_{r}\left(V_{0}\right)$. Let us denote by $P S(r)$ the multilinear part of $P S\left(V_{0}\right)$ of the $r$-homogeneous Schur functor $P S_{r}\left(V_{0}\right)$ for even space $V_{0}$ of dimension $r$. The space $P S(r)$ is a reducible $\mathfrak{S}_{r}$-module and from the decomposition of $P S\left(V_{0}\right)$ follows $P S(r) \cong \bigoplus_{\lambda \vdash r} S^{\lambda}$. The statement of the lemma follows by induction on the dimension $r$.

Now let us take $V$ to be a $m \mid n$-dimensional space. It is enough to apply the Schur functor $P S$ to the superspace $V$. The nontrivial $U\left(\mathfrak{g l}_{m \mid n}\right)$-modules $V^{\lambda}$ are labelled by Young diagrams within the $(m, n)$-hook and all these appear exactly once. Since $V^{\lambda} \equiv 0$ iff $\lambda \notin H(m, n)$ we get $P S(V) \cong \bigoplus_{\lambda \in H(m, n)} V^{\lambda}$.

Corollary 3.4. The hook generalization of the Schur identity reads

$$
\frac{\prod_{i<j, \hat{i} \neq \hat{j}}\left(1+x_{i} x_{j}\right)}{\prod_{i}\left(1-x_{i}\right) \prod_{i<j, \hat{i}=\hat{j}}\left(1-x_{i} x_{j}\right)}=\sum_{\lambda} h s_{\lambda}\left(x_{1}, \ldots, x_{m+n}\right)
$$

where $h s_{\lambda}(x)$ stands for the Hook Schur function of $m$ even and $n$ odd variables.

Proof. The $U\left(\mathfrak{g l}_{m \mid n}\right)$ character of the Schur module $V^{\lambda}, \lambda \in H(m, n)$ of the $m \mid n$ dimensional superspace is the hook Schur function [3]

$$
h s_{\lambda}\left(x_{1}, \ldots, x_{m+n}\right)=\sum_{\mu \subset \lambda} s_{\mu}\left(x_{1}, \ldots, x_{m}\right) s_{\lambda^{\prime} / \mu^{\prime}}\left(x_{m+1}, \ldots, x_{m+n}\right) .
$$

where $\lambda^{\prime}$ stands for the transposed Young diagram of $\lambda$. The hook Schur functions have also a combinatorial definition

$$
h s_{\lambda}(x)=\sum_{T \in S S Y T(\lambda)} x^{w t(T)} \quad \lambda \in H(m, n)
$$

where the sum runs over all $(m, n)$-semistandard Young tableaux of shape $\lambda$. One has $h s_{\lambda}(x)=0$ iff $\lambda \notin H(m, n)$.

The algebra $P S(V)$ is the universal enveloping algebra of the 2-nilpotent Lie superalgebra (6), hence the Poincaré-Bikhoff-Witt theorem for superalgebras yields

$$
P S(V) \cong \mathbb{S}\left(V \oplus \mathbb{S}^{1^{2}} V\right) \cong S\left(V_{0}\right) \otimes \Lambda\left(V_{1}\right) \otimes S\left(\Lambda^{2}\left(V_{0}\right)\right) \otimes S\left(S^{2}\left(V_{1}\right)\right) \otimes \Lambda\left(V_{0} \wedge V_{1}\right)
$$

Therefore the character $\chi_{P S(V)}(x)$ of the $U\left(\mathfrak{g l}_{m \mid n}\right)$-module $P S(V)$ reads

$$
\chi_{P S(V)}(x)=\frac{\prod_{i, \hat{i}=1}\left(1+x_{i}\right)}{\prod_{i, \hat{i}=0}\left(1-x_{i}\right)} \frac{\prod_{i<j, \hat{i} \neq \hat{j}}\left(1+x_{i} x_{j}\right)}{\prod_{i<j, \hat{i}=\hat{j}}\left(1-x_{i} x_{j}\right) \prod_{i: \hat{i}=1}\left(1-x_{i}^{2}\right)}
$$

which equals to the LHS of the eq. (10). On the other hand the character $\chi_{P S(V)}$ is a sum of the characters $h s_{\lambda}$ of the irreducible components $V^{\lambda}$ which ends the proof of the identity (10). 
4. Parastatistics Fock spaCes Revisited.

Parastatistics Fock space $\mathcal{F}(m \mid n ; p)$ of order $p$ is the quotient of the $P S$-algebra

$$
\mathcal{F}(m \mid n ; p)=P S(V) / M(V, p),
$$

where the graded ideal $M(V, p)$ is generated by the irreducible Schur module $V^{(p)}=$ $\mathbb{S}^{p+1} V \subset V^{\otimes p+1}$,

$$
M(V, p)=\left(\mathbb{S}^{p+1} V\right) .
$$

For parafermions $V=V_{0}$ and the space $\mathbb{S}^{p+1} V$ is the ordinary $p+1$-symmetrizer corresponding to the one row diagram

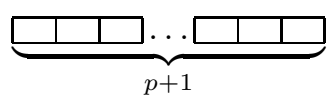

therefore in the Fock space $\mathcal{F}(m \mid 0 ; p)$ no more than $p$ identical paraparticles can occupy one state (tableau). Hence in general factoring by $\mathbb{S}^{p+1} V$ the parastatistics algebra $P S(V)$ is the superfication of the Fermi exculsion principle.

Definition 4.1. The S-submodule isomorphic to the factor of the $\mathfrak{S}$-module PS

$$
P S^{(p)} \cong P S /\left(S^{(p+1)}\right)
$$

such that $\mathcal{F}(m \mid n ; p) \cong P S^{(p)}(V)=\bigoplus_{r \geq 0} P S^{(p)}(r) \otimes_{\mathfrak{S}_{r}} V^{\otimes r}$ will be referred to as p-restricted parastatistics $\mathfrak{S}$-module

$$
P S^{(p)}=\bigoplus_{r \geq 0} P S^{(p)}(r) \quad P S^{(p)}(r) \subset \mathbb{C}\left[\mathfrak{S}_{r}\right] .
$$

The decomposition of the $p$-restricted $\mathfrak{S}$-module $P S^{(p)}$ contains once and exactly once each irreducible finite dimensional $\mathbb{C}\left[\mathfrak{S}_{r}\right]$-module $S^{\lambda}$ such that its partition $\lambda$ is restricted by $p \geq \lambda_{1} \geq \ldots \lambda_{r} \geq 0$,

$$
P S^{(p)}=\bigoplus_{\lambda: \lambda_{1} \leq p} S^{\lambda}
$$

which means that we eliminate from $P S$ all the irreducibles with diagrams with more than $p$ columns.

Let us denote by $F_{0}$ the set of the self-conjugated partitions $\eta=\eta^{\prime}$, i.e., the partitions $\eta$ with Frobenius notation

$$
\eta=\left(\alpha_{1}, \alpha_{2}, \ldots, \alpha_{r} \mid \alpha_{1}, \alpha_{2}, \ldots, \alpha_{r}\right), \quad \alpha_{1}>\alpha_{2}>\ldots>\alpha_{r} \geq 0,
$$

and by $F_{p}$ of the set of the partitions with $p$-augmented arms

$$
\eta_{(p)}=\left(\alpha_{1}+p, \alpha_{2}+p, \ldots, \alpha_{r}+p \mid \alpha_{1}, \alpha_{2}, \ldots, \alpha_{r}\right), \quad \alpha_{1}>\ldots>\alpha_{r} \geq 0 .
$$

Thus $\eta_{(p)} \in F_{p}$ when $\eta \in F_{0}$. One has $S^{\eta_{(p)}} \subset\left(S^{(p+1)}\right)$.

Corollary 4.2. The parastatistics Fock space $\mathcal{F}(m \mid n ; p)$ of order $p$ as a $U\left(\mathfrak{g l}_{m \mid n}\right)$ module is isomorphic to the sum of Schur modules

$$
\mathcal{F}(m \mid n ; p) \cong \bigoplus_{\lambda \in H^{(p)}(m, n)} V^{\lambda}
$$


where $H^{(p)}(m, n)$ stands for the set of hook partitions $H(m, n)$ with no more than $p$ columns, $\lambda_{1} \leq p$. The character formula for $\mathcal{F}(m \mid n ; p)$ yields the identity

$$
\frac{\sum_{\eta \in F_{0}}(-1)^{\frac{1}{2}(|\eta|+r)} h s_{\eta_{(p)}}(x) \prod_{i<j, \hat{i} \neq \hat{j}}\left(1+x_{i} x_{j}\right)}{\prod_{i}\left(1-x_{i}\right) \prod_{i<j, \hat{i}=\hat{j}}\left(1-x_{i} x_{j}\right)}=\sum_{\lambda: \lambda_{1} \leq p} h s_{\lambda}(x) .
$$

Proof. For an even space $V=V_{0}$ (i.e., parafermions only) the $U(\mathfrak{g l}(V))$-character $\chi_{P S^{(p)}(V)}$ of the parastatistics Fock space $P S^{(p)}(V) \cong \mathcal{F}(m \mid 0, p)$ reads

$$
\chi_{P S^{(p)}(V)}(x)=\sum_{\lambda: \lambda_{1} \leq p} s_{\lambda}(x)=\frac{\sum_{\eta \in F_{0}}(-1)^{\frac{1}{2}(|\eta|+r)} s_{\eta_{(p)}}(x)}{\prod_{i}\left(1-x_{i}\right) \prod_{i<j}\left(1-x_{i} x_{j}\right)} .
$$

Here the sum over the $p$-restricted Young diagrams is the character of $P S^{(p)}(V)$ and the sum over the diagrams from $E_{(p)}$ (13) is the character of the factor $P S(V) / M(V, p)$. In the works on parastatistics [15, 22] the proof of the identity (16) is attributed to R. King 1 . For our proof of the character identity see the appendix.

Now let $V$ be a superspace of dimension $m \mid n$. From the functoriality of the construction of the Fock space $\mathcal{F}(m \mid n ; p)$ as a submodule and factor-module of $P S(V)$

$$
\mathcal{F}(m \mid n ; p)=P S(V) / M(V, p) \cong P S^{(p)}(V)
$$

it follows the identity between the Hook Schur functions

$$
\frac{\sum_{\eta \in F_{0}}(-1)^{\frac{1}{2}(|\eta|+r)} h s_{\eta_{(p)}}(x)}{\sum_{\eta \in F_{0}}(-1)^{\frac{1}{2}(|\eta|+r)} h s_{\eta}(x)}=\sum_{\lambda: \lambda_{1} \leq p} h s_{\lambda}(x) .
$$

Then the formula for the $U\left(\mathfrak{g l}_{m \mid n}\right)$-character $\chi_{P S(V)}$ (10) implies the identity (15).

Remark. 4.3. The $N$-complexes [5] arise in a natural way in the approach of DuboisViolette and Marc Henneaux [6, 7] (see also the Marc Henneaux's contribution to this volume) to the higher gauge spin fields. In the study of the gauge $S$-spin fields (with $S \geq 1)$ the $(S+1)$-complexes are involved. The degrees of these complexes are spaces of tensors with Young-symmetry type with the constraint that the row lengths of the corresponding Young diagrams are no longer than the spin S. The components of the tensor fields on $\mathbb{R}^{D}$ are labelled by different Young tableaux (with entries from 1 to $D)$ and the total space of the $(S+1)$-complex has the same structure as the the parafermionic Fock space $\mathcal{F}(D \mid 0, S)$ of parastatistical order $S$.

\section{Deformed parastatistics and its para-Fock Space}

The parastatistics algebras (4) of creation and annihilation operators allow for $q$-deformations as introduced by Palev [21. The idea is to replace the universal enveloping algebra (UEA) $U\left(\mathfrak{o s p}_{1+2 m \mid 2 n}\right)$ by the quantum UEA $U_{q}\left(\mathfrak{o s p}_{1+2 m \mid 2 n}\right)$ written in an alternative form, with a system of relations between generators corresponding to the parastatistics creation and annihilation operators. We are going to describe the deformation $\mathcal{P S}(V)$ of the creation parastatistics algebra $P S(V)$. The space $\mathcal{P S}(V)$ is naturally a $U_{q}\left(\mathfrak{g l}_{m \mid n}\right)$-module and instead of working with the $U_{q}\left(\mathfrak{o s p}_{1+2 m \mid 2 n}\right)$ relations we choose another approach based on the $q$-Schur modules and the Hecke

\footnotetext{
${ }^{1}$ We are grateful to Ron King for sending us his proof of the identity (16).
} 
algebra. Our aim is to extract from $\mathcal{P} \mathcal{S}(V)$ a combinatorial algebra having as elements the super semistandard Young tableaux.

5.1. Hecke algebra. The Hecke algebra $H_{r}(q)$ is the associative algebra generated by $g_{1}, \ldots, g_{r-1}$ with the relations

$$
\begin{aligned}
g_{i} g_{i+1} g_{i} & =g_{i+1} g_{i} g_{i+1} & & i=1, \ldots, r-1 \\
g_{i} g_{j} & =g_{j} g_{i} & & |i-j| \geq 2 \\
g_{i}^{2} & =1+\left(q-q^{-1}\right) g_{i} & & i=1, \ldots, r-1
\end{aligned}
$$

The specialization $q=1$ yields the Coxeter relations of the symmetric group $\mathfrak{S}_{r}$ generated by the elementary transpositions $s_{i}=(i i+1)$ for $i=1, \ldots, r-1$.

The elements of $H_{r}(q)$ are indexed by permutations in $\sigma \in \mathfrak{S}_{r}, T_{\sigma} \in H_{r}(q)$ in the following way. Let $s_{i_{1}} \ldots s_{i_{k}}$ be the reduced word of the permutation $\sigma$, then

$$
T_{\sigma}:=T_{s_{i_{1}}} \ldots T_{s_{i_{k}}} \quad \sigma=s_{i_{1}} \ldots s_{i_{k}}
$$

where $T_{s_{i}}=g_{i}$ and the Coxeter relations (18) are equivalent to the relations

$$
\begin{aligned}
T_{\rho} T_{\sigma} & =T_{\rho \sigma} \quad \text { when } \quad|\rho \sigma|=|\rho|+|\sigma| \\
T_{s_{i}}^{2} & =1+\left(q-q^{-1}\right) T_{s_{i}}
\end{aligned}
$$

For $q$ generic the Hecke algebra $H_{r}(q)$ is isomorphic to the group algebra $\mathbb{C}\left[\mathfrak{S}_{r}\right]$. The irreducible $H_{r}(q)$-modules $\mathcal{H}^{\lambda}$ are indexed by Young diagrams with $r$ boxes $\lambda \vdash r$, i.e., in the same manner as the irreducible $\mathfrak{S}_{r}$-modules.

5.2. Parastatistics Hecke ideal. We now consider the $H_{3}(q)$-module $\mathcal{I}(3) \cong \mathcal{H}^{(2,1)}$ which is a deformation of the $\mathfrak{S}_{3}$-module $I(3)=e \mathbb{C}\left[\mathfrak{S}_{3}\right] \cong S^{(2,1)}$. To this end we find an idempotent $e(q) \in H_{3}(q)$ which is a deformation of the Eulerian idempotent $e$, in the sense that $e(1)=e$.

Let us denote by $\omega$ the maximal element in $H_{3}(q), \omega=g_{1} g_{2} g_{1}$.

Lemma 5.3. The equation for the idempotent $e(q) \in H_{3}(q)$

$$
\omega e(q)=e(q)
$$

fixes $e(q)$ completely when $q \neq \pm 1$. The unique solution is given by the expression

$$
\begin{aligned}
e(q) & :=\frac{1}{[3]}\left(T_{123}-\frac{1}{2}\left(T_{231}+T_{213}+T_{132}+T_{312}\right)+T_{321}\right) \\
& +\frac{q-q^{-1}}{2[3]}\left(T_{213}-T_{312}-T_{231}+T_{132}\right) .
\end{aligned}
$$

Proof. The statement can be checked by direct calculation in the Hecke algebra $H_{3}(q)$ taking into account $T_{123}=1, T_{213}=g_{1}, T_{132}=g_{2}, T_{213}=g_{1} g_{2}, T_{312}=g_{2} g_{1}, T_{321}=$ $g_{1} g_{2} g_{1}=\omega$.

Thus the symmetry $\omega e=e$ which holds true for the Eulerian idempotent $e$ in the group algebra $\mathbb{C}\left[\mathfrak{S}_{3}\right]$ is preserved during the deformation.

Let us define $\mathcal{I}(3)$ to be the right $H_{3}(q)$-ideal $\mathcal{I}(3)=e(q) H_{3}(q)$. By construction $\mathcal{I}(3)$ is an $H_{3}(q)$-ideal such that its "classical" limit $q \rightarrow 1$, i.e., its specialization to the $\mathbb{C}\left[\mathfrak{S}_{3}\right]$-module is $I(3)$. It is spanned by two elements $\boldsymbol{\Gamma}_{3}^{12}$ and $\boldsymbol{\Gamma}_{2}^{13}$

$$
\mathcal{I}(3)=\mathbb{C}(q) \Gamma_{3}^{12} \oplus \mathbb{C}(q) \Gamma_{2}^{13}
$$


which can be chosen to be

(24) $\Gamma_{2}^{13}=q\left(T_{213}-T_{231}\right)+T_{123}-T_{132}-T_{231}+T_{321}+q^{-1}\left(T_{312}-T_{132}\right)$,

(25) $\boldsymbol{\Gamma}_{3}^{12}=q\left(T_{132}-T_{312}\right)+T_{123}-T_{213}-T_{312}+T_{321}+q^{-1}\left(T_{231}-T_{213}\right)$.

Written in terms of the $H_{3}(q)$-generators $g_{1}$ and $g_{2}$ the basis of $\mathcal{I}(3)$ looks like

$$
\begin{aligned}
& \boldsymbol{\Gamma}_{2}^{13}=\mathbb{1}+q g_{1}-\left(1+q^{-1}\right) g_{2}-(1+q) g_{2} g_{1}+q^{-1} g_{1} g_{2}+g_{2} g_{1} g_{2}, \\
& \boldsymbol{\Gamma}_{3}^{12}=\mathbb{1}+q g_{2}-\left(1+q^{-1}\right) g_{1}-(1+q) g_{1} g_{2}+q^{-1} g_{2} g_{1}+g_{1} g_{2} g_{1},
\end{aligned}
$$

and the $H_{3}(q)$-action is determined by linear continuation of the actions

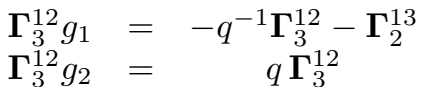

$$
\begin{aligned}
& \boldsymbol{\Gamma}_{2}^{13} g_{1}=q \boldsymbol{\Gamma}_{2}^{13} \\
& \boldsymbol{\Gamma}_{2}^{13} g_{2}=-q^{-1} \boldsymbol{\Gamma}_{2}^{13}-\boldsymbol{\Gamma}_{3}^{12} \text {. }
\end{aligned}
$$

5.4. Quantum Schur-Weyl Duality. We are now going to sketch how the Hecke algebra is related to the deformation $U_{q}\left(\mathfrak{g l}_{m \mid n}\right)$ of UEA $U\left(\mathfrak{g l}_{m \mid n}\right)$ providing a generalization of the Schur-Weyl duality. For the defining relations of the quantum UEA $U_{q}\left(\mathfrak{g l}_{m \mid n}\right)$ we send the reader to [2].

We need some preliminaries on the $R$-matrix.

Lemma 5.5. Let $V$ be a superspace of dimension $m \mid n$ over the field $K(q)$. The linear transformation $\hat{R} \in \operatorname{End}(V \otimes V)$ given by the $(m+n)^{2} \times(m+n)^{2}$ matrix

$$
\hat{R}_{k l}^{i j}=(-1)^{\hat{i} \hat{j}} q^{(-1)^{\hat{i}}} \delta_{i j} \delta_{l}^{i} \delta_{k}^{j}+\left(q-q^{-1}\right) \theta_{j i} \delta_{k}^{i} \delta_{l}^{j}
$$

is an $R$-matrix of the quantum linear supergroup $G L_{q}(m \mid n)$, i.e., $\hat{R}$ satisfies

i) the Yang-Baxter equation

$$
\hat{R}_{1} \hat{R}_{2} \hat{R}_{1}=\hat{R}_{2} \hat{R}_{1} \hat{R}_{2} \quad\left(\hat{R}_{1}=\hat{R} \otimes \mathbb{1} \quad \hat{R}_{2}=\mathbb{1} \otimes \hat{R}\right),
$$

ii) the Hecke relation

$$
\hat{R}^{2}=\mathbb{1}+\left(q-q^{-1}\right) \hat{R},
$$

having two eigenvalues $\pm q^{ \pm 1}$ appearing with multiplicities $\frac{m(m \pm 1)}{2}+\frac{n(n \mp 1)}{2}+m n$. The discrete step function $\theta_{i j}$ is 0 when $i<j$ and 1 when $i \geq j$.

5.6. Sign permutation action of the Hecke algeba $H_{r}(q)$. Let us consider the left action $\sigma_{q}: V^{\otimes r} \rightarrow V^{\otimes r}$ defined for the $H_{r}(q)$ generators by

$$
\pi_{q}\left(g_{s}\right) a_{i_{1}}^{\dagger} \ldots a_{i_{s}}^{\dagger} a_{i_{s+1}}^{\dagger} \ldots a_{i_{r}}^{\dagger}=\sum_{j_{s}, j_{s+1}} a_{i_{1}}^{\dagger} \ldots a_{j_{s}}^{\dagger} a_{j_{s+1}}^{\dagger} \ldots a_{i_{r}}^{\dagger} \hat{R}_{i_{s} i_{s+1}}^{j_{s} j_{s+1}} \quad s \leq r-1
$$

and extended by linearity. This action is indeed a $H_{r}(q)$-representation by virtue of the Lemma 5.5 and it will be referred to as sign permutation action of $H_{r}(q)$. In more details the action (28) reads

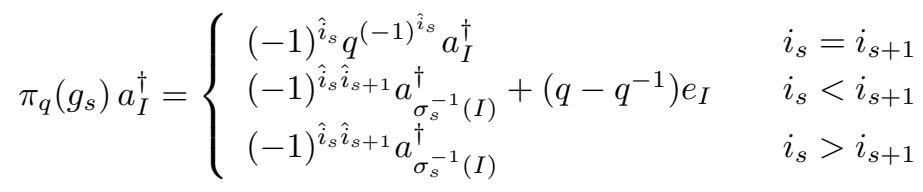

where the (multi)index permutation $\sigma_{s}^{-1}(I)=i_{1} \ldots i_{s+1} i_{s} \ldots i_{r}$. In the limit $q \rightarrow 1$ one retrieves the sign permutation action of the symmetric group,

$$
\left.u \otimes v \hat{R}\right|_{q \rightarrow 1}=(-1)^{\hat{u} \hat{v}} v \otimes u .
$$


Theorem 5.7. (quantum Schur-Weyl duality [19]) The sign permutation action $\pi_{q}$ of the Hecke algebra $H_{r}(q)$ and the action $\rho$ of the quantum UEA $U_{q}\left(\mathfrak{g l}_{m \mid n}\right)$ on $V^{\otimes r}$ are centralizers to each other

$$
\rho\left(U_{q}\left(\mathfrak{g l}_{m \mid n}\right)\right)=\operatorname{End}_{H_{r}(q)}\left(V^{\otimes r}\right), \quad \pi_{q}\left(H_{r}(q)\right)=\operatorname{End}_{U_{q}\left(\mathfrak{g l}_{m \mid n}\right)}\left(V^{\otimes r}\right) .
$$

The quantum version of the Schur-Weyl duality between the quantum UEA $U_{q}\left(\mathfrak{g l}_{m}\right)$ action on the tensor power of the vector representation $V^{\otimes r}$ and the permutation action of the Hecke algebra $H_{r}(q)$ is due to Jimbo [11, whereas its super-counterpart given by Theorem 5.7 was done by Mitsuhashi [19.

5.8. The $q$-Schur functor. The quantum Schur-Weyl duality stated in Theorem 5.7 allows to build the representations of the quantum UEA $U_{q}\left(\mathfrak{g l}_{m \mid n}\right)$ from the Hecke modules in the same fashion as the representations of $U\left(\mathfrak{g l}_{m \mid n}\right)$ (and $G L(m \mid n)$ ) are built from $\mathfrak{S}$-modules.

Let us have $H$-module $\mathcal{M}$ which is a family of right $H_{r}(q)$-modules $\mathcal{M}(r), r \geq 0$. Its associated $q$-Schur functor $\mathcal{M}: g V e c t \rightarrow g V e c t$ is defined as

$$
\mathcal{M}(V):=\bigoplus_{r \geq 0} \mathcal{M}(r) \otimes_{H_{r}(q)} V^{\otimes r}
$$

where $V$ is a superspace over the field $K(q)$ and the $H_{r}(q)$-action on $V^{\otimes r}$ is the sign permutation action $\pi_{q}(28)$. The image $\mathcal{M}(V)$ carries the structure of $U_{q}\left(\mathfrak{g l}_{m \mid n}\right)$ module. The homogeneous components of $\mathcal{M}(V)$ are denoted by

$$
\mathcal{M}_{r}(V):=\mathcal{M}(r) \otimes_{H_{r}(q)} V^{\otimes r}, \quad \mathcal{M}(V)=\bigoplus_{r \geq 0} \mathcal{M}_{r}(V),
$$

and their irreducible $U_{q}\left(\mathfrak{g l}_{m \mid n}\right)$-submodules are denoted by

$$
V^{\lambda}=\mathcal{H}^{\lambda} \otimes_{H_{r}(q)} V^{\otimes r}, \quad \lambda \in H(m, n) .
$$

Definition 5.9. The braided parastatistics superalgebra $\mathcal{P S}(V)$ is the factor algebra of the tensor algebra of $V$ by the ideal $\mathcal{I}(V)$

$$
\mathcal{P S}(V)=T(V) / \mathcal{I}(V)
$$

where $\mathcal{I}(V)=\bigoplus_{r \geq 3} \mathcal{I}_{r}(V)$ is the twosided ideal generated by $\mathcal{I}_{3}(V)$

$$
\mathcal{I}_{r}(V)=\sum_{i+j+3=r} V^{\otimes i} \otimes \mathcal{I}_{3}(V) \otimes V^{\otimes j}, \quad r \geq 3 .
$$

and $\mathcal{I}_{3}(V)$ stands for the image of the right $H_{3}(q)$-module $\mathcal{I}(3)=e(q) H_{q}(3)$ by the $q$-Schur functor

$$
\mathcal{I}_{3}(V)=\mathcal{I}(3) \otimes_{H_{3}(q)} V^{\otimes 3} .
$$

Proposition 5.10. (17]) Let $a_{i}^{\dagger}$ be a basis of the $m \mid n$-dimensional superspace $V=$ $\oplus \mathbb{C}(q) a_{i}^{\dagger}$. The superspace $\mathcal{I}_{3}(V) \cong V^{(2,1)}$ is an irreducible $U_{q}\left(\mathfrak{g l}_{m \mid n}\right)$-module

$$
\mathcal{I}_{3}(V)=\bigoplus_{\substack{i_{1} i_{2} \\ i_{3}}} \mathbb{C}(q) \Gamma_{i_{3}}^{i_{1} i_{2}}
$$


where the sum runs over all $(m, n)$-Semistandard Young Tableaux of shape $(2,1)$ and the spanning elements (chosen to be polynomial in $q^{-1}$ ) read

$$
\begin{aligned}
& \Gamma_{i_{2}}^{i_{1} i_{3}}:=\quad \llbracket a_{i_{2}}^{\dagger}, \llbracket a_{i_{3}}^{\dagger}, a_{i_{1}}^{\dagger} \rrbracket \rrbracket_{q^{-2}}+q^{-1} \llbracket a_{i_{3}}^{\dagger}, \llbracket a_{i_{1}}^{\dagger}, a_{i_{2}}^{\dagger} \rrbracket \rrbracket \quad i_{1}<i_{2}<i_{3}, \\
& \Gamma_{i_{3}}^{i_{1} i_{2}}:=\quad \llbracket \llbracket a_{i_{3}}^{\dagger}, a_{i_{1}}^{\dagger} \rrbracket, a_{i_{2}}^{\dagger} \rrbracket_{q^{-2}}+q^{-1} \llbracket \llbracket a_{i_{2}}^{\dagger}, a_{i_{3}}^{\dagger} \rrbracket, a_{i_{1}}^{\dagger} \rrbracket \quad i_{1}<i_{2}<i_{3}, \\
& \Gamma_{i_{2}}^{i_{1} i_{2}}:=\quad \llbracket \llbracket a_{i_{1}}^{\dagger}, a_{i_{2}}^{\dagger} \rrbracket, a_{i_{2}}^{\dagger} \rrbracket_{q^{-1}} \quad i_{1}<i_{2}, \quad \hat{i}_{2}=1, \\
& \Gamma_{i_{2}}^{i_{1} i_{2}}:=\quad \llbracket a_{i_{2}}^{\dagger}, \llbracket a_{i_{1}}^{\dagger}, a_{i_{2}}^{\dagger} \rrbracket \rrbracket_{q^{-1}} \quad i_{1}<i_{2}, \quad \hat{i}_{2}=0, \\
& \Gamma_{i_{3}}^{i_{2} i_{2}}:=\quad \llbracket a_{i_{2}}^{\dagger}, \llbracket a_{i_{2}}^{\dagger}, a_{i_{3}}^{\dagger} \rrbracket \rrbracket_{q^{-1}} \quad i_{2}<i_{3}, \quad \hat{i}_{2}=1, \\
& \Gamma_{i_{2}}^{i_{2} i_{3}}:=\quad \llbracket \llbracket a_{i_{2}}^{\dagger}, a_{i_{3}}^{\dagger} \rrbracket, a_{i_{2}}^{\dagger} \rrbracket_{q^{-1}} \quad i_{2}<i_{3}, \quad \hat{i}_{2}=0 .
\end{aligned}
$$

Proof. The Schur functor attached to the $H_{3}(q)$-module $\mathcal{I}(3)=\mathbb{C}(q) \boldsymbol{\Gamma}_{3}^{12} \oplus \mathbb{C}(q) \boldsymbol{\Gamma}_{2}^{13}$ is the $U_{q}\left(\mathfrak{g l}_{m \mid n}\right)$-module $\mathcal{I}_{3}(V)$ of the relations of the algebra $\mathcal{P} \mathcal{S}(V)$. The direct calculation of the quantities $\Gamma_{3}^{12} \otimes_{H_{3}(q)}\left(a_{i}^{\dagger} \otimes a_{j}^{\dagger} \otimes a_{k}^{\dagger}\right)$ and $\boldsymbol{\Gamma}_{2}^{13} \otimes_{H_{3}(q)}\left(a_{i}^{\dagger} \otimes a_{j}^{\dagger} \otimes a_{k}^{\dagger}\right)$ yields elements which are either proportional (with coefficients in $\mathbb{C}(q)$ ) to some $\Gamma_{k}^{i j}$ from (33) or zero. By construction these elements span $\mathcal{I}_{3}(V)$.

\section{Plactic monoid.}

Let us consider the free monoid of words written in the alphabet $A$, the multiplication being the juxtaposition of words. The plactic monoid [14] is the set of the equivalence classes in the free monoid for the equivalence defined as the words with $P$-equivalent tableaux in the Robinson-Schensted $(P, Q)$-correspondence. The $P$-equivalence coincides with the equivalence with respect to the Knuth relations

$$
\begin{array}{ll}
x z y=z x y & x \leq y<z \\
y x z=y z x & x<y \leq z
\end{array}
$$

with $x, y, z \in A$. The classes of equivalent words in the plactic monoid are in bijection with the Semistandard Young Tableaux with entries from the alphabet $A$. The algebra of the plactic monoid will be denoted as $\operatorname{Plac}(A)$.

On the other hand in view of Theorem 3.2 and Corollary 3.4 the states in the parastatistics algebra $P S(V)$ with $m$ parafermi degrees of freedom are in bijection with the Semistandard Young Tableau with entries from $\{1, \ldots, m\}$, that is, the set of indices of the vector space $V=V_{0}$.

This parallel suggests an interrelation between the algebra $P S(V)$ and the plactic algebra $\operatorname{Plac}(V)$. Surprisingly in revealing this interrelation the quantum UEAs play a key role [4].

\section{Plactic Superalgebra and the Parastatistics Algebra}

Let $K$ be the subring of rational functions without a pole at $q^{-1}, K \subset \mathbb{C}(q)$. By the evaluation map $f\left(q^{-1}\right) \mapsto f(0)$ we have an isomorphism $K / q^{-1} K \cong \mathbb{C}$.

Definition 7.1. (Kashiwara 12 ) Let $W$ be a $\mathbb{C}(q)$-vector space. The local base of $W$ at $q^{-1}=0$ is the pair $(L, B)$ where $L$ is a free $K$-module and $B$ is a base of the $\mathbb{C}$-vector space $L / q^{-1} L$. 
Let us denote by $\Gamma$ the basis of the space $\mathcal{I}_{3}(V)$,

$$
\Gamma=\left\{\begin{array}{lll}
\Gamma_{i_{3}}^{i_{1} i_{2}} & \mid i_{i_{3}} i_{1} i_{2}
\end{array} \text { is a }(m, n) \text {-semistandard hook tableau }\right\}
$$

and by $\gamma$ the base of $\Gamma / q^{-1} \Gamma$.

By applying the evaluation map one gets the following

Corollary 7.2. The base $\gamma$ is given by the elements

$$
\begin{array}{llll}
a_{i_{1}}^{\dagger} a_{i_{3}}^{\dagger} a_{i_{2}}^{\dagger}-(-1)^{\hat{i}_{1} \hat{i}_{3}} a_{i_{3}}^{\dagger} a_{i_{1}}^{\dagger} a_{i_{2}}^{\dagger}, & \left(i_{1} \leq i_{2}<i_{3}, \hat{i}_{2}=0\right) & \text { or } & \left(i_{1}<i_{2} \leq i_{3}, \hat{i}_{2}=1\right) \\
a_{i_{2}}^{\dagger} a_{i_{1}}^{\dagger} a_{i_{3}}^{\dagger}-(-1)^{\hat{i}_{1} \hat{i}_{3}} a_{i_{2}}^{\dagger} a_{i_{3}}^{\dagger} a_{i_{1}}^{\dagger}, & \left(i_{1}<i_{2} \leq i_{3}, \hat{i}_{2}=0\right) & \text { or } \quad\left(i_{1} \leq i_{2}<i_{3}, \hat{i}_{2}=1\right)
\end{array}
$$

The $U_{q}\left(\mathfrak{g l}_{m \mid n}\right)$-module $\mathcal{I}_{3}(V)$ has a local basis $(\Gamma, \gamma)$ at the point $q^{-1}=0$.

The algebra $\mathcal{P S}(V)$ at the point $q^{-1}=0$ with relations

$$
\begin{aligned}
& x z y=(-1)^{\hat{x} \hat{z}} z x y, \quad(x \leq y<z, \hat{y}=0) \quad \text { or } \quad(x<y \leq z, \hat{y}=1) \\
& y x z=(-1)^{\hat{x} \hat{z}} y z x, \quad(x<y \leq z, \hat{y}=0) \quad \text { or } \quad(x \leq y<z, \hat{y}=1)
\end{aligned}
$$

will be denoted by $\operatorname{Plac}_{\mathbb{Z}_{2}}(V)$, a super-counterpart of the plactic algebra. These relations are a $\mathbb{Z}_{2}$-version of the Knuth relations 2 of the plactic monoid on a signed alphabet $\{1, \ldots m, \overline{1}, \ldots, \bar{n}\}$ of the indices of the basis of the superspace $V=V_{0} \oplus V_{1}$. The classes of equivalent words modulo the super-Knuth relations are in one-to-one correspondence with the semistandard $(m, n)$-hook tableaux on one hand and and to the states in the universal parastatistics algebra $\mathcal{P S}(V)$ on the other hand.

The states in the parastatistics Fock space $\mathcal{F}(m \mid n, p)$ correspond to semistandard $(m, n)$-hook tableaux whose rows are $p$-restricted, that is, with lengths not exceeding $p$ boxes. In the superalgebra $\operatorname{Plac}_{\mathbb{Z}_{2}}(V)$ the $p$-restriction on the rows is imposed by the condition

$$
x_{1} \ldots x_{k} y_{k+1} \ldots y_{p+1}=0 \quad x_{1} \leq \ldots \leq x_{k}<y_{k+1}<\ldots<y_{p+1}
$$

where $\hat{x}_{i}=0$ and $\hat{y}_{j}=1$.

\section{ACKNOWLEDGMENTS}

It's a pleasure to thank Michel Dubois-Violette for his constant interest and encouragement. He had first the idea to draw a parallel between the parastatistics algebra and the plactic monoid. Todor Popov is indebted to Boyka Aneva, Oleg Ogievetsky, Tchavdar Palev, Neli Stoilova and Joris Van der Jeugt for many enlightening discussions. T.P. thanks for the hospitality of the Institut de Recherche Mathématique Avancée, Strasbourg and Centre de Physique Théorique, Luminy and acknowledges partial support from the project GIMP No.ANR-05-BLAN-0029-01 of the Agence Nationale pour la Recherche and the Bulgarian National Foundation for Scientific Research (contract Ph-1406).

\footnotetext{
${ }^{2}$ The super-Knuth relations obtained in the work [13] are the same up the sign depending on the $\mathbb{Z}_{2}$-grading.
} 


\section{Appendix A. Parafermionic Fock Space Character}

We are giving a sketch of the proof of the character identity

$$
\sum_{\eta \in F_{p}}(-1)^{\frac{1}{2}(|\eta|-(p-1) r)} s_{\eta}(x)=\prod_{i}\left(1-x_{i}\right) \prod_{i<j}\left(1-x_{i} x_{j}\right) \sum_{\lambda: l\left(\lambda^{\prime}\right) \leq p} s_{\lambda}(x)
$$

which is equivalent to the one in eq. (15) when we change the summation on the self-conjugated partitions $F_{0}$ by summation on the $p$-augmented partitions $F_{p}$. In Macdonald's book on symmetric functions the special case $p=0$ of the latter formula is given (see p.79 [18])

$$
\sum_{\eta \in F_{0}}(-1)^{\frac{1}{2}(|\eta|+r)} s_{\eta}(x)=\prod_{i}\left(1-x_{i}\right) \prod_{i<j}\left(1-x_{i} x_{j}\right)
$$

where the sum is over the selfconjugated partitions $\eta=\eta^{\prime}$.

We shall prove the character identity for every $p \in \mathbb{N}$ with the help of the Weyl identity for the Weyl groups $W$ of type $A_{n-1}$ and $B_{n}$

$$
\sum_{w \in W} \varepsilon(w) e^{w \rho}=\prod_{\alpha \in R^{+}}\left(e^{\frac{\alpha}{2}}-e^{-\frac{\alpha}{2}}\right)
$$

where $\varepsilon(w)$ is the sign of the element $w$ and $\rho$ is the Weyl vector $\rho=\frac{1}{2} \sum_{\alpha \in R^{+}} \alpha$. The exponentials of the weights are formal.

Let $v_{i}$ be the standard basis of $\mathbb{R}^{n}$. The root system of type $A_{n-1}$ and $B_{n}$ are

$$
\Delta_{0}=\left\{\mp v_{i} \pm v_{j}\right\} \quad \subset \quad \Delta=\left\{ \pm v_{i}, \pm v_{i} \pm v_{j}, \mp v_{i} \pm v_{j}\right\} \quad 1 \leq i<j \leq n
$$

and the subsystems of the positive and negative roots are $\Delta_{0}^{ \pm}=\Delta_{0} \cap \Delta^{ \pm}$and

$$
\Delta^{+}=\left\{v_{i}, v_{i} \pm v_{j}\right\}, \quad \Delta^{-}=\left\{-v_{i},-v_{i} \pm v_{j}\right\}, \quad 1 \leq i<j \leq n .
$$

hence the respective Weyl vectors differ by a constant vector $\theta=\frac{1}{2} \sum v_{i}$

$$
\rho_{0}=\sum_{i=1}^{n}\left(\frac{n}{2}+\frac{1}{2}-i\right) v_{i}, \quad \rho=\sum_{i=1}^{n}\left(n+\frac{1}{2}-i\right) v_{i}, \quad \rho=n \theta+\rho_{0} .
$$

The Weyl group $W_{0}$ of the root system $\Delta_{0}$ is the symmetric group $\mathfrak{S}_{n}$ permuting the indexes of the vectors $v_{i}$, while the Weyl group $W$ of the root system $\Delta$ is the semidirect product of $W_{0}$ with the group $\mathbb{Z}_{2}^{n}$ acting by $v_{i} \mapsto \epsilon_{i} v_{i}$ where $\epsilon_{i}= \pm$.

The root system $\Delta$ has two commuting involutions $c_{0}$ and $c$

$$
c_{0}: v_{i} \mapsto v_{i^{\prime}}:=v_{n+1-i}, \quad c: v_{i} \mapsto-v_{i}
$$

uniquely determined by $c_{0}\left(\Delta_{0}^{+}\right)=\Delta_{0}^{-}$and $c\left(\Delta^{+}\right)=\Delta^{-}$. These involutions are defined by the action of the element of the maximal length in the Weyl group of $A_{n-1}$ and $B_{n}$, respectively.

The Weyl identity for $\Delta^{+}$yields

$$
\sum_{w \in W} \varepsilon(w) e^{w \rho}=e^{\rho} \prod_{i}\left(1-e^{-v_{i}}\right) \prod_{i<j}\left(1-e^{-v_{i}-v_{j}}\right) \prod_{i<j}\left(1-e^{-v_{i}+v_{j}}\right)
$$

The sum over the Weyl group $W$ can be split as

$$
\sum_{\left\{\epsilon_{i}\right\} \in \mathbb{Z}_{2}^{n}} \sum_{w_{0} \in \mathfrak{S}_{n}}(-1)^{\epsilon} \varepsilon\left(w_{0}\right) e^{w_{0} \epsilon_{i} \rho}=e^{n \theta} \sum_{\left\{\epsilon_{i}\right\}} \sum_{w_{0} \in \mathfrak{S}_{n}}(-1)^{\epsilon} \varepsilon\left(w_{0}\right) e^{w_{0} \rho_{0}-w_{0}\left(\sum\left(1-\epsilon_{i}\right) \rho_{i} v_{i}\right)}
$$


where $\varepsilon\left(w_{0}\right)$ is the signature of the permutation $w_{0}$ and $(-1)^{\epsilon}:=\prod \epsilon_{i}=(-1)^{\sum \frac{1-\epsilon_{i}}{2}}$.

The action of the involution $c_{0}$ is equivalent to a resummation on $W_{0}$ and change the sum by the signature of the element of maximal length in $W_{0}$, i.e., $(-1)^{\frac{n(n-1)}{2}}$.

With the identification $x_{i}=e^{-v_{i}}$ and using the Weyl identity for $\Delta_{0}^{+}$we get

$$
\prod_{i}\left(1-x_{i}\right) \prod_{i<j}\left(1-x_{i} x_{j}\right)=\sum_{\left\{\epsilon_{i}\right\}}(-1)^{\epsilon} \frac{\left|x_{j}^{\mu_{i}}\right|}{\left|x_{j}^{\rho_{0 i}}\right|} \quad \text { with } \quad \mu_{i}=\rho_{0 i}+\left(1-\epsilon_{i^{\prime}}\right) \rho_{i^{\prime}} .
$$

Lemma A.1. In the $\mathfrak{S}_{n}$-orbit of the weight $\mu=\mu\left(\epsilon_{1}, \ldots, \epsilon_{n}\right)=\sum \mu_{i} v_{i}$ uniquely determined by the data of $\left\{\epsilon_{1}, \ldots, \epsilon_{n}\right\}$ there exists one and only one representative

$$
\lambda+\rho_{0}=\sigma(\mu) \quad \text { such that } \quad \lambda_{1} \geq \lambda_{2} \geq \ldots \geq \lambda_{n} \geq 0,
$$

i.e., $\lambda$ is a Young diagram. The weight $\lambda$ is autoconjugated $\lambda=\lambda^{\prime}$. The signature of the permutation $\sigma$ is

$$
\varepsilon(\sigma)=(-1)^{\frac{1}{2}\left(\sum|\lambda|-r\right)},
$$

where $|\lambda|=\sum \lambda_{i}$ and $r$ is the number of diagonal boxes in $\lambda$.

Proof: By construction the numbers $\mu_{i}$ are all different therefore $\mu_{i}$ can be always arranged by a permutation $\sigma$ into decreasing order, and then it will be a sum of a partition $\lambda$ and the vector $\rho_{0}$ (with strictly decreasing components $\rho_{0 i+1}-\rho_{0 i}=1$ ).

Given a vector $\mu=\mu\left(\epsilon_{1}, \ldots, \epsilon_{n}\right)$, that is, a configuration $\left\{\epsilon_{1}, \ldots, \epsilon_{n}\right\}$ we choose $\lambda$ to be such that the projector $\frac{1-\epsilon_{i}}{2}$ projects to the hook $\left(\beta_{i} \mid \alpha_{i}\right)$ in the Frobenius notation with $\alpha_{i}=\beta_{i}=n-i$. In other words, $\lambda=\left(\alpha_{1}, \ldots, \alpha_{r} \mid \alpha_{1}, \ldots \alpha_{r}\right)$ and $r=\sum \frac{1-\epsilon_{i}}{2}$. From the formula for the number of boxes of $\lambda$

$$
|\lambda|=\sum \mu_{\sigma(i)}-\sum \rho_{0 i}=\sum_{i} \rho_{i}\left(1-\epsilon_{i}\right)=\sum_{i}(2 n-2 i+1) \frac{1-\epsilon_{i}}{2}
$$

it follows that our choice of $\lambda$ is a compatible one and therefore is the only possible one since $\lambda$ such that $\lambda+\rho_{0}=\sigma(\mu)$ is unique.

For example the $2^{2}$ Young diagrams $\lambda$ appearing in the case $n=2$ read
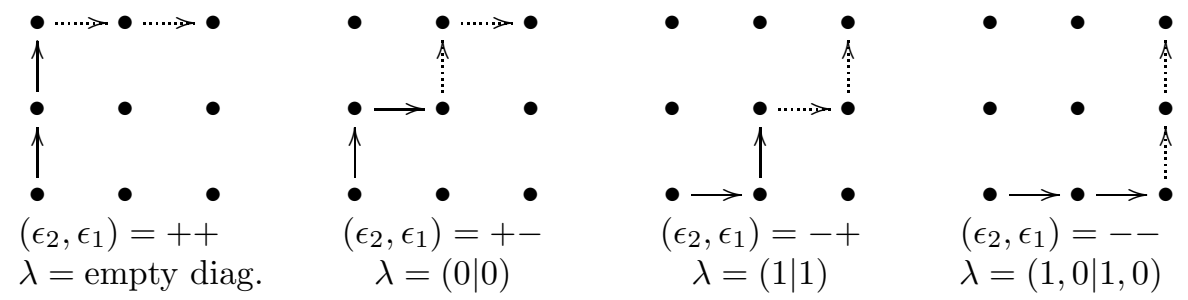

The signature of $\sigma$ is the number of exchanges needed to bring the components $\mu_{i}=\rho_{0 i}+\left(1-\epsilon_{i^{\prime}}\right) \rho_{i^{\prime}}$ into decreasing order,

$$
\begin{aligned}
\varepsilon(\sigma) & =\prod_{i<j} \operatorname{sgn}\left(\mu_{i}-\mu_{j}\right)=\prod_{i<j} \operatorname{sgn}\left(\left(1-\epsilon_{i^{\prime}}\right) \rho_{i^{\prime}}-\left(1-\epsilon_{j^{\prime}}\right) \rho_{j^{\prime}}\right) \\
& =\prod_{i>j} \operatorname{sgn}\left(\left(1-\epsilon_{i}\right) \rho_{i}-\left(1-\epsilon_{j}\right) \rho_{j}\right):=\prod_{i>j} s_{i j} .
\end{aligned}
$$

Due to $\rho_{i}<\rho_{j}$ when $i>j$ the exchange sign $s_{i j}$ depends only on the smaller index

$$
s_{i j}=\operatorname{sgn}\left(\left(1-\epsilon_{i}\right) \rho_{i}-\left(1-\epsilon_{j}\right) \rho_{j}\right)=(-1)^{\frac{1-\epsilon_{j}}{2}}, \quad i>j
$$


hence the signature of the permutation $\sigma$ depends only on $|\lambda|$ and $r$

$$
\varepsilon(\sigma)=\prod_{i>j} s_{i j}=(-1)^{\sum_{j}(n-j) \frac{1-\epsilon_{j}}{2}}=(-1)^{\frac{1}{2}(|\lambda|-r)} .
$$

Using the lemma and the determinantal formula $s_{\lambda}(x)\left|x_{j}^{\rho_{0 i}}\right|=\left|x_{j}^{\rho_{0 i}+\lambda_{i}}\right|$ we get

$$
\prod_{i}\left(1-x_{i}\right) \prod_{i<j}\left(1-x_{i} x_{j}\right)=\sum_{\left\{\epsilon_{i}\right\}}(-1)^{\epsilon} \frac{\left|x_{j}^{\mu_{i}}\right|}{\left|x_{j}^{\rho_{0 i}}\right|}=\sum_{\lambda \in F_{0}}(-1)^{\frac{1}{2}(|\lambda|+r)} s_{\lambda}(x)
$$

where the sum runs on the autoconjugated Young diagrams $\left(\mathfrak{g l}_{n}\right.$-weights). This ends the proof of the character identity in the special case $p=0$.

The sum over the Schur functions with no more than $p$ columns can be represented as a quotient of determinants (see p.84 in the book of Macdonald [18])

$$
\sum_{\lambda: l\left(\lambda^{\prime}\right) \leq p} s_{\lambda}\left(e^{-v_{1}}, \ldots, e^{-v_{n}}\right)=e^{-p \theta} D_{\rho+p \theta} / D_{\rho}
$$

where $D_{\rho}=\sum_{w \in W} \varepsilon(w) e^{w \rho}$. The character formula (34) of the para-Fock module can be rewritten into the following equivalent form

$$
\sum_{\eta \in F_{p}}(-1)^{\frac{1}{2}(|\eta|-(p-1) r)} s_{\eta}(x)=(-1)^{\left(\begin{array}{c}
n \\
2
\end{array}\right)} \frac{e^{-(n+p) \theta} D_{\rho+p \theta}}{\left|x_{j}^{\rho_{0 i}}\right|} .
$$

Proceeding as in the case $p=0$ we bring the RHS to

$$
\sum_{\left\{\epsilon_{i}\right\}}(-1)^{\epsilon} \frac{\left|x_{j}^{\nu_{i}}\right|}{\left|x_{j}^{\rho_{0 i}}\right|} \quad \text { with } \quad \nu_{i}=\rho_{0 i}+\left(1-\epsilon_{i^{\prime}}\right) \rho_{i^{\prime}}+\frac{p}{2} .
$$

Lemma A.2. In the $\mathfrak{S}_{n}$-orbit of the weight $\nu=\nu\left(\epsilon_{1}, \ldots, \nu_{n}\right)=\sum \nu_{i} v_{i}$ there exists one and only one representative $\lambda$ such that

$$
\lambda+\rho_{0}=\sigma(\nu), \quad \lambda_{1} \geq \ldots \geq \lambda_{n} \geq 0,
$$

belongs to $F_{p}$, that is, $\lambda=\left(\alpha_{1}+p, \ldots, \alpha_{r}+p \mid \alpha_{1}, \ldots, \alpha_{r}\right)$ with $r \leq n$. The signature of the permutation $\sigma$ is

$$
\varepsilon(\sigma)=(-1)^{\frac{1}{2}(|\lambda|-(p+1) r)} .
$$

The $2^{n}$ configurations of $\left\{\epsilon_{i}\right\}$ are in bijection with the $\mathfrak{g l}_{n}$-weights from $F_{p}$.

Sketch of the proof: The proof goes in the same lines as the proof of lemma A.1 Given a collection $\left\{\epsilon_{i}\right\}$ we choose a diagram $\lambda$ such that the projector $\frac{1-\epsilon_{i}}{2}$ projects on the Frobenius hook $\left(\beta_{i} \mid \alpha_{i}\right)$ with $\beta_{i}=n-i+p$ and $\alpha_{i}=n-i$. From the formula for the number of boxes in $\lambda \in F_{p}$

$$
|\lambda|=\sum\left(\nu_{i}-\rho_{0 i}\right)=\sum \rho_{i}\left(1-\epsilon_{i}\right)+\frac{p}{2}=\sum(2 n-2 i+1+p) \frac{1-\epsilon_{i}}{2},
$$

implies $\lambda+\rho_{0}=\sigma(\nu)$ (such a $\lambda$ is unique). Every configuration $\left\{\epsilon_{i}\right\}$ gives a different Young diagram $\lambda \in F_{p}$ thus the total number is $2^{n}$. The signature $\varepsilon(\sigma)$ is

$$
\varepsilon(\sigma)=\prod_{i<j} \operatorname{sgn}\left(\nu_{i}-\nu_{j}\right)=(-1)^{\sum(n-j) \frac{1-\epsilon_{j}}{2}}=(-1)^{\frac{1}{2}(|\lambda|-(p+1) r)} .
$$


Finally with the help of the lemma we conclude that

$$
\sum_{\left\{\epsilon_{i}\right\}}(-1)^{\epsilon} \frac{\left|x_{j}^{\nu_{i}}\right|}{\left|x_{j}^{\rho_{0 i}}\right|}=\sum_{\lambda \in F_{p}}(-1)^{r}(-1)^{\frac{1}{2}(|\lambda|-(p+1) r)} \frac{\left|x_{j}^{\rho_{0 i}+\lambda}\right|}{\left|x_{j}^{\rho_{0 i}}\right|}=\sum_{\lambda \in F_{p}}(-1)^{\frac{1}{2}(|\lambda|-(p-1) r)} s_{\lambda}(x)
$$

so the character identity (34) of the parastatistics Fock space holds true.

\section{REFERENCES}

[1] B. Aneva and T. Popov, Hopf Structure and Green Ansatz of Deformed Parastatistics Algebras, J. Phys. A: Math. Gen. 38 (2005), 6473-6484.

[2] G. Benkart, S.-J. Kang and M. Kashiwara, Crystal Bases for the Quantum Superalgebra $U_{q} \mathfrak{g l}(m, n)$, Journal of AMS 13 (2000), 295-331.

[3] A. Berele and A. Regev, Hook Young Tableaux with Application to Combinatorics and to Representations of Lie Superalgebras. Adv. Math. 64 (1987), 118-175.

[4] E. Date, M. Jimbo and T. Miwa, Representations of $U_{q}(\mathfrak{g l}(n, \mathbb{C}))$ at $q=0$ and the RobinsonSchensted correspondence, in "Physics and Mathematics of Strings", Memorial Volume of Vadim Knizhnik. eds. L. Brink, D. Friedan, A. M. Polyakov (World Sci. Publ., Teaneck, NJ, 1990), 185211.

[5] M. Dubois-Violette, $d^{N}=0$ : Generalized homology. K-Theory 14 (1998), 371-404.

[6] M. Dubois-Violette and M. Henneaux, Generalized cohomology of irreducible tensor fields of mixed Young-symmetry type. Lett. Math. Phys. 49 (1999), 245-252.

[7] M. Dubois-Violette and M. Henneaux, Tensor fields of mixed Young symmetry type and $N$ complexes. Commun. Math. Phys. 226 (2002), 393-418.

[8] M. Dubois-Violette and T. Popov, Homogeneous algebras, statistics and combinatorics. Lett. Math. Phys. 61 (2002), 159-170.

[9] H. S. Green, A Generalized Method of Field Quantization. Phys. Rev. 90 (1953), 270-273.

[10] R. Fioresi, Tensor representations of the General Linear Super Group. math/0601555

[11] M. Jimbo, A $q$-analogue of $U(\mathfrak{g l}(N+1))$, Hecke algebra and the Yang-Baxter equation. Lett. Math. Phys. 11 (1986), 247-252.

[12] M. Kashiwara, On crystal bases. Representations of Groups, Proceedings of the 1994 Annual Seminar of the Canadian Math. Soc. Ban16 (1995) 155-197, Amer. Math. Soc., Providence, RI.

[13] R. La Scala, V. Nardozza, D. Senato, Super RSK-Algorithms and super plactic monoid. Int. Journal of Algebra and Computation 16 (2006), 377-396.

[14] A. Lascoux, M.P. Schützenberger, Le monö̈de plaxique. Quaderni de "La ricerca scientifica" 109, Roma, CNR (1981) 129-156.

[15] S. Lievens, N. I. Stoilova and J. Van der Jeugt, The paraboson Fock space and unitary irreducible representations of the Lie superalgebra osp $(1 \mid 2 n)$. Commun. Math. Phys. 281(2008), 805-826.

[16] J.-L. Loday, Série de Hausdorff, idempotents eulériens et algèbres de Hopf. Exposition. Math. 12 (1994), 165-178.

[17] J.-L. Loday and T. Popov, Algebras of Young Tableaux and Parastatistics. Proceedings of the VII International Workshop "Lie Theory and Its Applications in Physics", eds. H.-D. Doebner and V.K. Dobrev,(Heron Press, Sofia, 2008), 423-430.

[18] I.G. Macdonald, Symmetric functions and Hall polynomials, Oxford, 1979.

[19] H. Mitsuhashi, Schur-Weyl Reciprocity between the Quantum Superalgebra and the IwahoriHecke Algebra, Algebras and Representation Theory 9 (2006), 309-322.

[20] T. Palev, Para-Bose and para-Fermi operators as generators of orthosymplectic Lie superalgebras, Journal of Math. Phys. 23 (1982), 1100-1102.

[21] T. Palev, A $q$-Deformation of the Parastatistics and an Alternative to the Chevalley Description of $U_{q}(\operatorname{osp}(2 n+1 / 2 m))$, Commun. Math. Phys.196 (1998), 429-443.

[22] N. I. Stoilova and J. Van der Jeugt, Parafermions, parabosons and representations of so $(\infty)$ and $\operatorname{osp}(1 \mid \infty)$, arXiv:0801.3909 1. 
Institut de Recherche Mathématique Avancée, CNRS et Université de Strasbourg, 7 Rue R. Descartes, 67084 Strasbourg Cedex, France

E-mail address: loday@math.u-strasbg.fr

$U R L$ : www-irma.u-strasbg.fr/ loday/

Institute for Nuclear Research and Nuclear Energy,, Bulgarian Academy of Sciences, Bld. Tsarigradsko chaussée 72, BG-1784 Sofia, Bulgaria

E-mail address: tpopov@inrne.bas.bg 